\title{
Topological Insulator to Dirac Semimetal Transition Driven by Sign Change of Spin-Orbit Coupling in Thallium Nitride
}

\author{
Xian-Lei Sheng, ${ }^{1}$ Zhijun Wang, ${ }^{1}$ Rui Yu, ${ }^{2}$ Hongming Weng, ${ }^{1,3}$, Zhong Fang, ${ }^{1,3}$ and Xi Dai ${ }^{1,3}$ \\ ${ }^{1}$ Beijing National Laboratory for Condensed Matter Physics, \\ and Institute of Physics, Chinese Academy of Sciences, Beijing 100190, China \\ ${ }^{2}$ International Center for Materials Nanoarchitectonics (WPI-MANA), \\ National Institute for Materials Science, Tsukuba 305-0044, Japan \\ ${ }^{3}$ Collaborative Innovation Center of Quantum Matter, Beijing, China
}

(Dated: October 11, 2018)

\begin{abstract}
Based on the first-principles calculations, we reveal that TIN, a simple binary compound with Wurtzite structure, is a three-dimensional (3D) topological insulator (TI) with effectively negative spin-orbit coupling $\lambda_{\text {eff }}<0$, which makes it distinguished from other TIs by showing opposite spin-momentum locking effect in its surface states. The sign of $\lambda_{\text {eff }}$ depends on the hybridization between $\mathrm{N}-2 p$ and $\mathrm{Tl}-5 d$ states, and can be tuned from negative to postive by lattice strain or chemical substitution, which drive the system into a Dirac semimetal with 3D Dirac cones in its bulk states. Such topological phase transition can be realized by electronic mechanism without breaking any crystal symmetry.

PACS numbers: 71.20.-b, 73.20.-r, 31.15.A-
\end{abstract}

Introduction. The spin-orbit coupling (SOC) plays important roles in generating topologically non-trivial band structure. [1 5] For examples, in graphene [6] (or other similar materials like silicene [7]), the SOC opens a gap and makes it a $2 \mathrm{D}$ TI, while in $\mathrm{Bi}_{2} \mathrm{Se}_{3}$ family [8] 3D TIs, both the band inversion and gap opening are caused by strong SOC. For the design and optimization of topological electronic materials, it is therefore highly desirable to have tunable SOC. From the viewpoint of atomic physics, this goal is hardly achieved, because the strength of SOC for each atomic orbital is almost predetermined by the type of atoms (namely the atomic number). In a solid state compound, however, we are interested in the effective SOC of certain Bloch states, which in general compose of multiple atomic orbitals with different SOC. This complication, on the other hand, gives us a chance to tune the SOC effectively. Not only the strength but also the sign of SOC can be effectively tuned through the proper manipulating of the orbital characters for low energy Bloch states, which generates interesting topological phase transition as we will addressed in the paper.

It has been indicated in literatures that several rare compounds 911 may have effectively negative SOC. For example, in $\mathrm{HgS}$, it was suggested that the SOC splitting for the $p$-orbitals around the valence band top (i.e, the $\Gamma$ point) is opposite to that of the usual semiconductors with Zinc-blende structure. In other words, the $j=1 / 2$ doublet states is energetically higher than the $j=3 / 2$ quartet states in $\mathrm{HgS}$, resulting in a TI state rather than the zero-gap semimetal state in $\mathrm{HgSe}$ and $\mathrm{HgTe}$. This proposal is interesting, unfortuanately, the existence of band inversion in $\mathrm{HgS}$ is challenged by the recent $\mathrm{GW}$ calculations $[12$ and is not confirmed experimentally [13]. In this paper, we will demonstrate that Wurtzite TIN [14] is a promising TI with "negative" SOC. What makes it unique from other TIs is its opposite spin-momentum locking effect, which can be readily observed experimentally. Furthermore, the sign of effective SOC in TIN can be reversed by suitable lattice strain without breaking any crystal symmetry, which leads a topological phase transition from a TI to a Dirac semimetal with 3D Dirac cones in its bulk. [15-17] All of these make TIN a valuable playground for quantum manipulation.

Mechanism for "negative" SOC. In Zinc-blende IIIV or II-VI semiconductors, such as GaAs or CdTe, the conduction band minimum at $\Gamma$ labeled as $\Gamma_{6}$ is mostly from cation $s$-orbital, while the valence band maximum is mostly from anion $p$-orbitals, which split into $\Gamma_{8}\left(j=\frac{3}{2}\right)$ and $\Gamma_{7}\left(j=\frac{1}{2}\right)$ manifolds in the presence of SOC. The band gap $E_{g}$ is defined as the energy difference between $\Gamma_{6}$ and $\Gamma_{8}$ states, $E_{g}=E_{\Gamma_{6}}-E_{\Gamma_{8}}$, and similarly, the effective SOC is defined as $\lambda_{e f f}=E_{\Gamma_{8}}-E_{\Gamma_{7}}$. Both $E_{g}$ and $\lambda_{e f f}$ are positive for GaAs and CdTe, while it is known that $E_{g}$ is negative in $\mathrm{HgTe}$ (i.e, the $s$-like band is lower than the $p$-like bands) leading to a topologically nontrivial state. Unfortunately, this is a zero gap semimetal state (rather than a true insulator) due to the four fold degeneracy of $\Gamma_{8}$ protected by the cubic symmetry of the lattice. On the other hand, if both $E_{g}$ and $\lambda_{e f f}$ are negative as suggested for $\mathrm{HgS}$, the $\Gamma_{7}$ states, which are only two-fold degenerated, will be higher than the $\Gamma_{8}$ states, and the band inversion between the $\Gamma_{6}$ and $\Gamma_{7}$ states will lead to a TI. Simultaneous requirements of both $E_{g}<0$ and $\lambda_{e f f}<0$ therefore make the possible material realization difficult.

To get $\lambda_{\text {eff }}<0$, a mechanism is illustrated as the following, by takeing the Wurtzite $\mathrm{ZnO}$ as a example. [18, 19] $\mathrm{ZnO}$ is a normal semiconductor with $E_{g}>$ 0 , however, its valence band top shows negative SOC $\left(\lambda_{\text {eff }}<0\right)$, mostly due to the presence of shallow core 
$3 d$ states of $\mathrm{Zn}$. In $\mathrm{ZnO}$, the $\mathrm{Zn}$ atoms are under approximately tetrahedra crystal field of $\mathrm{O}$, which leads to sizable $t_{2 g}$ and $e_{g}$ splitting of the Zn-3d orbitals (with the $t_{2 g}$ states energetically higher than the $e_{g}$ states). We ignore the $e_{g}$ states, and concentrated on the $t_{2 g}$ manifold, which mainly consists of three atomic orbitals, $d_{x y}, d_{y z}$ and $d_{z x}$ orbitals. Considering the SOC effect among the three $t_{2 g}$ orbitals (i.e, projection of SOC matrix into the subspace spanned by these three orbitals), it is interesting to note that the SOC is effectively negative. [20] As the results, the $t_{2 g}$ states will further split into a $j_{\text {eff }}=1 / 2$ doublet and a $j_{e f f}=3 / 2$ quartet states with the former energetically higher. This is in opposite to the SOC splitting among the $p$ orbitals. If the $t_{2 g}$ are shallow enough, they will hybridize with the O- $2 p$ states significantly, and the valence band top should be composed of both $2 p$ and $3 d$ characters. Since the SOC among the O- $2 p$ and Zn- $t_{2 g}$ atomic orbitals have opposite sign, the final effective SOC of the valence band top therefore must be determined by its relative composition, and can be tuned by controlling the $p-d$ hybridization in the system. For $\mathrm{ZnO}$, in particular, the positive SOC splitting among O- $2 p$ orbitals are relatively smaller, the large $3 d$ components of the valence band top leads to the effectively negative SOC (Fig 1 ).

Crystal structure and Methods. TIN was synthesized in 1974 and it has Wurtzite structure 14] (as shown in Fig 2(a)) with experimental lattice parameters $a=3.68 \AA$ and $c=6.01 \AA$. The Wyckoff position of $\mathrm{N}$ is $(1 / 3,2 / 3,0)$ and that of $\mathrm{Tl}$ is $(1 / 3,2 / 3,0.381)$. Wurtzite structure has the $\cdots \mathrm{AB}-\mathrm{AB}-\mathrm{AB} \cdots$ stacking sequence along (0001) direction, and each primitive unit cell contains two formula units. Both $\mathrm{Tl}$ and $\mathrm{N}$ are tetrahedrally coordinated with slightly hexagonal distortion. Our firstprinciples calculations has been performed by using the WIEN2K package 21] with the full potential linearized augmented plane wave method (FLAPW). The generalized gradient approximation (GGA) is used for the exchange correlation potential. Brillouin zone (BZ) integration with a mesh of $13 \times 13 \times 7$ sampling is used. The muffin-tin radii $\left(R_{M T}\right)$ are chosen as 2.11 bohr both for $\mathrm{Tl}$ and $\mathrm{N}$. The maximum size of the plane wave vector $\left(K_{\max }\right)$ is determined by $K_{\max } R_{M T}=7.0$. For the later analysis, we construct the maximally localized Wannier functions (MLWF) 22 for $\mathrm{Tl} s$ and $\mathrm{N} p$ orbitals, which reproduce the low energy bands nicely. The MLWF was then used for the effective Hamiltonian of both bulk and semi-infinite surface.

Electronic structure. The band structures of TIN are calculated without and with SOC included, respectively, as shown in Fig 2 (c) and (d). The bands from -6 to $4 \mathrm{eV}$ are mostly coming from the N-2p and Tl- $6 s$ states. From the partial density of states (DOS), it is easy to find that Tl-6s state around the $\Gamma$ point $\left(\Gamma_{7}^{s}\right)$ is lower than $\mathrm{N}-2 p$ by about $2.0 \mathrm{eV}$ for both cases of with and without SOC. This suggests that TIN is a material with band inversion (i.e., $E_{g}<0$ ), similar to the case of HgTe. Considering

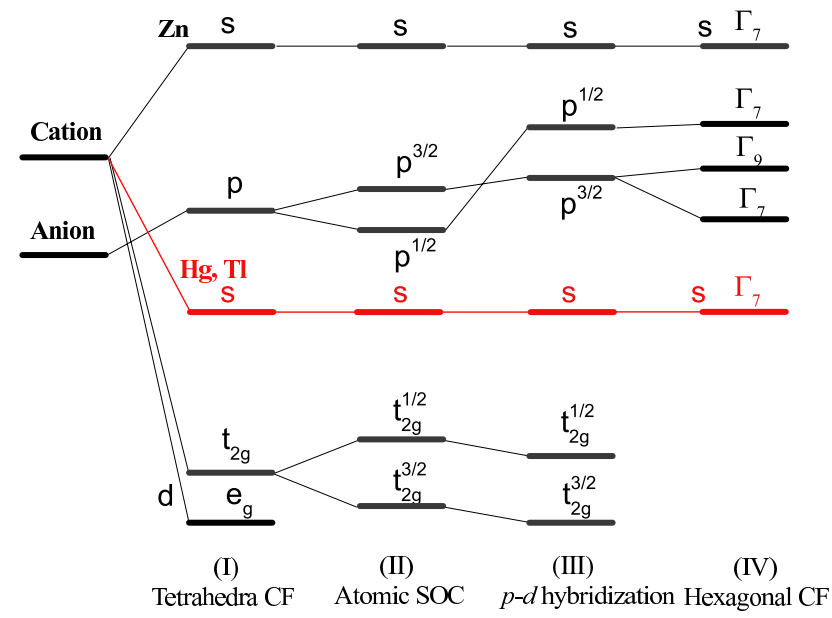

FIG. 1: (Color online) The energy level diagram of Wurtzite semiconductor at $\Gamma$ by taking into account of (I) idea tetrahedral crystal field (CF), (II) atomic spin-orbit coupling (SOC), (III) $p$ - $d$ hybridization and (IV) hexagonally distorted CF in real structure. The $s$ orbital of $\mathrm{Zn}$ in $\mathrm{ZnO}$ is higher than anion $p$-orbitals, while that of $\mathrm{Hg}$ in $\mathrm{HgTe}$ and $\mathrm{HgS}$ or $\mathrm{Tl}$ in $\mathrm{TlN}$ might be lower and result in inverted band structure.

the possible underestimation of band gap by the GGA, we further check the band inversion by using modified Becke-Jonhson potential 23] and HSE method 24], respectively, and still find band inversion of about $1.5 \mathrm{eV}$, which is very large compared to most known TIs. Thus, we conclude that the band inversion in TIN is robust and is not an artifact of GGA type calculation.

The Tl $5 d$-orbitals are fully occupied and locate mainly around $-10.0 \mathrm{eV}$ below the Fermi level. They are relatively extended with a sizable band width of about 2.5 $\mathrm{eV}$. There are apparently quite much $5 d$ DOS contribution to the $\mathrm{N}-2 p$ bands around the Femi level, which indicates remarkable $p$ - $d$ hybridization. This situation is quite similar to that in $\mathrm{ZnO}$ and leads to effective "negative" SOC for the states around the valence band top. Due to the reduced symmetry (the slightly hexagonal distortion), the $\Gamma_{8}\left(j=\frac{3}{2}\right)$ states should further split into $\Gamma_{9}^{\frac{3}{2}}$ and $\Gamma_{7}^{\frac{3}{2}}$ manifolds. Finally, as shown in Fig 2 (d), the valence $2 p$ band top in TIN split into three groups: $\Gamma_{9}^{\frac{3}{2}}$, $\Gamma_{7}^{\frac{3}{2}}$ and split-off band $\Gamma_{7}^{\frac{1}{2}}$. We find that their energies descend in the order of $\Gamma_{7}^{\frac{1}{2}}, \Gamma_{9}^{\frac{3}{2}}$ and $\Gamma_{7}^{\frac{3}{2}}$, which sugests $\lambda_{\text {eff }}<0$. Thus, TIN satisfies both the conditions of $E_{g}<0$ and $\lambda_{e f f}<0$, and should be a 3D topological insulator with negative SOC. Our calculations indeed found that it is a semiconductor with the direct (indirect) band gap of $36 \mathrm{meV}(25 \mathrm{meV})$ and $25 \mathrm{meV}$. Since TIN has no inversion symmetry, the Wilson loop method is employed to study the evolution of hybrid one-dimensional Wannier function center 25,26 , and its $\mathbb{Z}_{2}$ topological indices are found to be $(1 ; 000)$ (see Supplemental Material for details). 
(a)
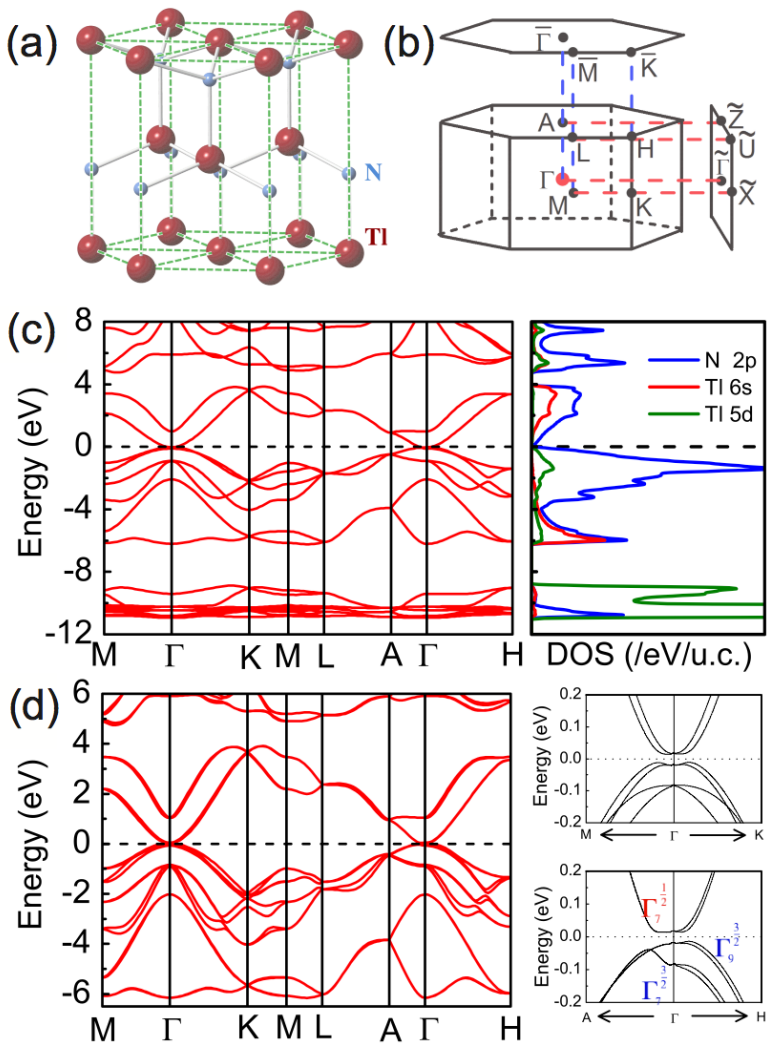

FIG. 2: (Color online) (a) Crystal structures of Wurtzite TIN and (b) its Brillouin zone (BZ), as well as the projected surface BZ of (0001) and (0100) surfaces.(c) The band structure and projected density of states of Wurtzite TIN without SOC. (d) The band structure with SOC and partially enlarged view around $\Gamma$ point.

3D strong TI supports odd number of Dirac-cone-like surface states, which should have characteristic spinmomentum locking effect. Almost all of the presently known TIs have shown left-handed spin-momentum locking when Fermi level is above the Dirac point. 8, 25, 27, 28. As shown in Fig, 3 . TIN does have single Diraccone-like surface states around $\bar{\Gamma}$ for both (0001) and (0100) surfaces, while the spin-momentum locking has right-handed helicity, in opposite to all other known TIs up to now. It would be very interesting to check this by spin-resolved ARPES experiments. It is also fundamentally interesting to see what will happen in the interface formed by two TIs with opposite spin-momentum locking helicity. [1] Another plausible remarkable thing is when $s$-wave superconducting pairing interaction is introduced to them by proximity effect, [29] the induced topological superconducting state will have opposite chirality, being $p+i p$ for left-handed one and $p$-ip for right-handed one. Heterostructure of them might have more fascinat-
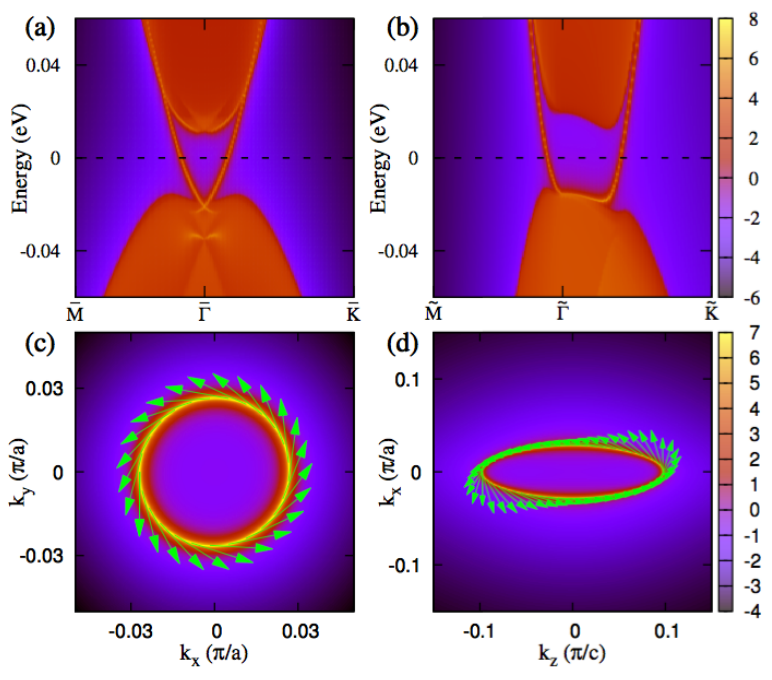

FIG. 3: (Color online)The calculated surface states (upper panels) and corresponding Fermi surfaces and spin textures (lower panels) of Wurtzite TIN on (0001) (left panels) and (0100) (right panels) surface, respectively.

ing quantum phenomena.

$p$-d hybridization. As mentioned above, the $p-d$ hybridization [30] is the origin of "negative" SOC splitting in $p$ orbitals. To explicitly reveal the underlying physics, an effective Hamiltonian with the basis of $\mathrm{N} 2 p$ and $\mathrm{Tl} 5 d$ orbitals is established. Here the Tl- $s$ orbital and the crystal field due to hexagonal distortion are neglected since they have negligible effect on the effective SOC splitting in $p$ orbitals. Due to the tetrahedra crystal field, only $t_{2 g}$ orbitals can have the hybridization with $p$ orbitals and $e_{g}$ part is ignored. For both $p$ and $t_{2 g}$ orbitals, their atomic SOC is firstly considered in their own subspace. The spin-orbit splitting of $t_{2 g}$ is found to be opposite to that of $p$ states with $D_{\frac{1}{2}}\left(j=\frac{1}{2}\right)$ higher than $D_{\frac{3}{2}}\left(j=\frac{3}{2}\right)$.

Based on this, we can further include the $p$ - $d$ hybridization, parameterized as $t_{p d}$, to see its influence on the effective spin-orbit spitting in $p$ orbitals. Only the $P$ and $D$ basis with the same representation can have nonzero hybridization, i.e., $\left\langle P_{j}, j_{z}|H| D_{j^{\prime}}, j_{z}^{\prime}\right\rangle=t_{p d} \delta_{j, j^{\prime}} \delta_{j_{z}, j_{z}^{\prime}}$. Such a simplified model, in the basis set of $\left|P_{j}, j_{z}\right\rangle$ and $\left|D_{j}, j_{z}\right\rangle$ (with $j=\frac{3}{2}, \frac{1}{2}$ and $j_{z}=-j,-j+1, \cdots, j-1, j$ ), can be 

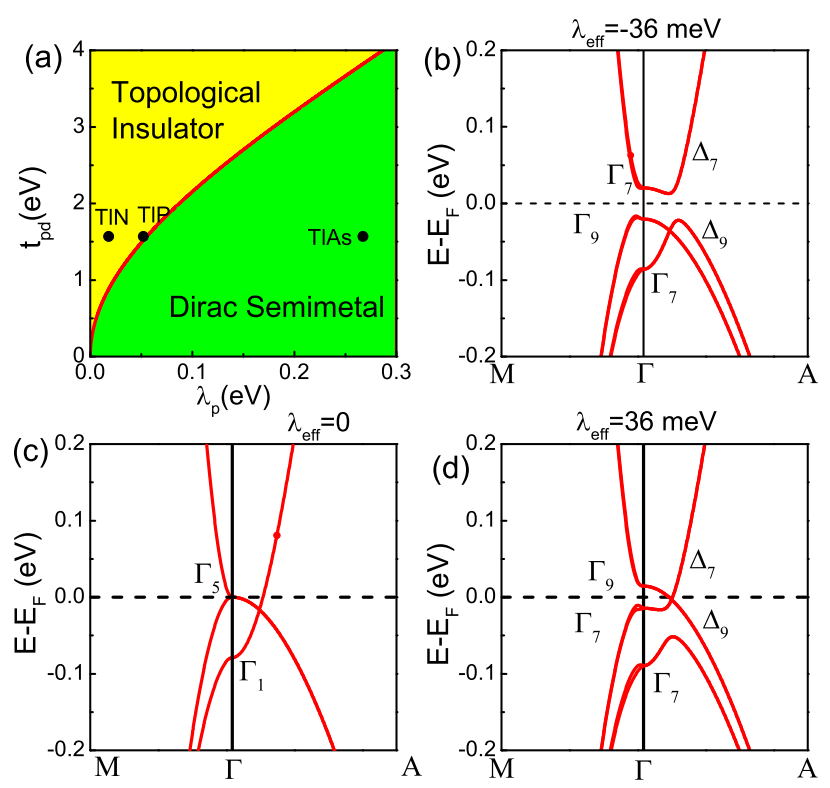

FIG. 4: (Color online) (a) Phase diagram of topological insulator and topological 3D Dirac semimetal depending on $p$ - $d$ hybridization $\left(t_{p d}\right)$ and atomic SOC of $p$ orbital $\left(\lambda_{p}\right)$. The band structure with (b) $\lambda_{e f f}=-36 \mathrm{meV}$, (c) $\lambda_{e f f}=0 \mathrm{meV}$ and (d) $\lambda_{\text {eff }}=-36 \mathrm{meV}$.

written in second quantized form,

$$
\begin{array}{r}
H_{p d}=\sum_{j, j_{z}} E_{j j_{z}}^{p} \hat{c}_{j, j_{z}}^{\dagger} \hat{c}_{j, j_{z}}+\sum_{j, j_{z}} E_{j j_{z}}^{d} \hat{d}_{j, j_{z}}^{\dagger} \hat{d}_{j, j_{z}} \\
+\sum_{j, j_{z}}\left[t_{p d} \hat{c}_{j, j_{z}}^{\dagger} \hat{d}_{j, j_{z}}+t_{p d}^{*} \hat{d}_{j, j_{z}}^{\dagger} \hat{c}_{j, j_{z}}\right]
\end{array}
$$

where $\hat{c}_{j, j_{z}}\left(\hat{d}_{j, j_{z}}\right)$ and $\hat{c}_{j, j_{z}}^{\dagger}\left(\hat{d}_{j, j_{z}}^{\dagger}\right)$ are the electron annihilation and creation operators at orbital $\left|P_{j}, j_{z}\right\rangle\left(\left|D_{j}, j_{z}\right\rangle\right)$. The diagonal term of $P$ orbitals $E_{j j_{z}}^{p}=\frac{\lambda_{p}}{3}$ if $j=\frac{3}{2}$, and $E_{j j_{z}}^{p}=-\frac{2 \lambda_{p}}{3}$ if $j=\frac{1}{2}$. The diagonal term of $t_{2 g}$ orbitals $E_{j j_{z}}^{d}=E_{d}-\frac{\lambda_{d}}{3}$ if $j=\frac{3}{2}$, and $E_{j j_{z}}^{d}=E_{d}+\frac{2 \lambda_{d}}{3}$ if $j=\frac{1}{2}$. The parameter $\lambda_{p}$ and $\lambda_{d}$ is the SOC parameters for $\mathrm{N}$ $2 p$ and $\mathrm{Tl} 5 d$, respectively. $E_{d}$ is the on-site energy of $5 d$ orbitals and that of $p$ is taken as zero.

For the Hamiltonian, Tl-5d SOC could be obtained by FLAPW calculation as $\lambda_{d}=2.168 \mathrm{eV}$, and $E_{d}$ takes the value of $-10.0 \mathrm{eV}$, which is approximately the gravity center of $\mathrm{Tl} 5 d$-orbitals in Fig2 (c) and (d). Depending on the strength of $p$ - $d$ hybridization $t_{p d}$ and $\lambda_{p}$, the system falls into two different phases, as shown in Fig 4 (a). In the strong $p$ - $d$ hybridization limit, the effective SOC $\lambda_{e f f}$ of anion's $p$ orbitals is negative and dominated by the SOC of cation's $5 d$ orbitals. The $p$ states split into a $j_{\text {eff }}=1 / 2$ doublet and a $j_{\text {eff }}=3 / 2$ quartet states with the former energetically higher. The band structure opens a gap in the whole BZ, and the system is a TI. In the strong $\lambda_{p}$ limit, $\lambda_{e f f}$ is dominated by the atomic SOC of $p$ orbitals, and it is positive. The $j_{\text {eff }}=1 / 2$ doublet states is energetically lower than the $j_{\text {eff }}=3 / 2$ quartet states, leading the system in a topological semimetal rather than a true insulator. Between the two regions, there should exist a critical line with $\lambda_{e f f}=0$. In other words, $t_{p d}$ and $\lambda_{p}$ are in balance. For material realization, TIN and $\mathrm{HgS}$ are at TI region, TlAs and HgTe are at TSM region, and TIP is almost at the borderline.

Topological phase transition by tuning effective SOC. As SOC plays the critical role in determining the topology of bands, tuning its strength and sign can introduce nontrivial topological phase transition. To show how this can happen in realistic material $\mathrm{TIN}$, an effective ab initio tight-binding Hamiltonian base on MLWFs of Tl- $6 s$ and $\mathrm{N}-2 p$ orbitals has been established. The effective SOC $\lambda_{e f f}$ is then added onto N-2p MLWFs as a tunable parameter. As shown in Fig 4(b), taking $\lambda_{e f f}=-36 \mathrm{meV}$ can reproduce the GGA+SOC calculation very well and TIN is a TI. When $\lambda_{\text {eff }}$ is zero, this reproduces the GGA calculation, the band inversion and the double degeneracy of $p_{x}$ and $p_{y}$ orbitals lead to zero gap semimetal state, as shown in Fig 4 (c). When $\lambda_{\text {eff }}$ is taken as $36 \mathrm{meV}$, it becomes a topological 3D Dirac semimetal with Dirac point on the path $\Gamma$-A. The sign reversal of $\lambda_{e f f}$ leads to the energy order reversal of $\Gamma_{9}^{\frac{3}{2}}$ and $\Gamma_{7}^{\frac{1}{2}}$ states. When $\Gamma_{7}^{\frac{1}{2}}$ is higher $\left(\lambda_{e f f}<0\right)$, the inverted $\Gamma_{7}^{s}$ can have hybridization with it in all direction of $\mathrm{BZ}$ and it is TI. When $\Gamma_{9}^{\frac{3}{2}}$ is higher $\left(\lambda_{e f f}>0\right), \Gamma_{7}^{s}$ bands exactly cross $\Gamma_{9}^{\frac{3}{2}}$ at the Dirac point since they belong to $\Delta_{7}$ and $\Delta_{9}$ irreducible representation, respectively, which are distinguished by the $\mathrm{C}_{3}$ rotation symmetry along $\Gamma$-A. Such band inversion resulted 3D Dirac semimetal state protected by crystal symmetry is the similar as those in $\mathrm{Na}_{3} \mathrm{Bi}$ and $\mathrm{Cd}_{3} \mathrm{As}_{2}$. [16, 17] We have found that when lattice strain is of $a=1.06 a_{0}$ and $c=1.10 c_{0}\left(a_{0}\right.$ and $c_{0}$ are experimental lattice constants), the band structure from first-principles calculation is the same as that in Fig.4(d) and TIN becomes a 3D Dirac semimetal (see Supplemental Material for details). 3D Dirac semimetal is a symmetry-protected topological state with a single pair of 3D Dirac points in the bulk and non-trivial Fermi arcs on the surfaces. The 3D Dirac point can be described as four-component Dirac fermions, which can be viewed as two copies of distingct Weyl fermions. Therefore, Weyl semimetal could be realized based on 3D Dirac semimetal when time-reversal or inversion symmetry was broken. [16, 17, 31] It can also be driven into TI by symmetry breaking, and quantum spin Hall effect in its quantum well structure.

In summary, we have shown that TIN is a $3 \mathrm{D}$ TI with effectively "negative" SOC. The band inversion between Tl- $6 s$ and N- $2 p$ orbital is robust and the $p$ - $d$ hybridization between $\mathrm{N} 2 p$ and $\mathrm{Tl} 5 d$ determines its effective SOC. Its surface state has right-handed helical spin- 
momentum locking, which is opposite to other known TIs. Heterostructure formed by two TIs with opposite spin-momentum locking helicity might host novel phenomena. The effective SOC can be tuned to be positive by suitable lattice strain without breaking any crystal symmetry and drives TIN from TI to 3D Dirac semimetal. These make TIN quite unique and a good playground for further study.

The author XLS acknowledges helpful discussions with L. R. Shaginyan, Fei Ye and Jian-Zhou Zhao. HMW acknowledges the hospitality during his stay in NICHe, Tohoku University. We acknowledge the supports from the NSF of China and the 973 program of China (No. 2011CBA00108 and 2013CB921700).

* Electronic address: hmweng@iphy.ac.cn

[1] C. L. Kane, E. J. Mele, Phys. Rev. Lett. 95, 146802 (2005).

[2] B. A. Bernevig, S. C. Zhang, Phys. Rev. Lett. 96, 106802 (2006).

[3] J. E. Moore and L. Balents, Phys. Rev. B 75, 121306 (2007).

[4] L. Fu, C. L. Kane, and E. J. Mele, Phys. Rev. Lett. 98, 106803 (2007).

[5] L. Fu and C. L. Kane, Phys. Rev. B 76, 045302 (2007).

[6] C. L. Kane, E. J. Mele, Phys. Rev. Lett. 95, 226801 (2005).

[7] C.-C. Liu, W. Feng and Y. Yao Phys. Rev. Lett. 107, 076802 (2011).

[8] H. Zhang, C. X. Liu, X. L. Qi, X. Dai, Z. Fang, S.-C. Zhang, Nature Phys. 5, 438 (2009); Y. Xia, et.al., Nature Phys. 5, 398 (2009); Y. L. Chen, et.al., Science 325, 178 (2009).

[9] J. Vidal, X. Zhang, V. Stevanović, J. W. Luo, and A. Zunger, Phys. Rev. B 86, 075316 (2012).

[10] F. Virot, R. Hayn, M. Richter, and J. van den Brink, Phys. Rev. Lett. 106, 236806 (2011).

[11] Qingze Wang, Shu-chun Wu, Claudia Felser, Binghai Yan, and Chao-Xing Liu, arXiv:1404.7091 (2014).

[12] A. Svane, N. E. Christensen, M. Cardona, A. N. Chantis, M. van Schilfgaarde, and T. Kotani, Phys. Rev. B 84, 205205 (2011).

[13] M. Cardona, R. K. Kremer, R. Lauck, G. Siegle, A. Muñoz, and A. H. Romero, Phys. Rev. B, 80, 195204 (2009).

[14] A. N. Pilyankevich, L. R. Shaginyan, and A. F. Andreeva, Bull. Russ. Acad. Sci, Phys. Ser. 38,163 (1974); A. F. Andreeva, A. N. Pilyankevich and L. R. Shaginyan, Izv. Akad. Mauk SSSR Ser. Fiz. 38, 1537 (1974).

[15] S. M. Young, S. Zaheer, J. C. Y. Teo, C. L. Kane, E. J. Mele, and A. M. Rappe, Phys. Rev. Lett., 108, 140405 (2012).

[16] Z. J. Wang, Y. Sun, X. Q. Chen, C. Franchini, G. Xu, H. M. Weng, X. Dai, and Z. Fang, Phys. Rev. B 85, 195320 (2012); Z. K. Liu, B. Zhou, Y. Zhang, Z. J. Wang, H. M. Weng, D. Prabhakaran, S.-K. Mo, Z. X. Shen, Z. Fang, X. Dai, Z. Hussain, Y. L. Chen, Science 343, 864 (2014).

[17] Z. J. Wang, H. M. Weng, Q. S. Wu, X. Dai, and Z. Fang,
Phys. Rev. B 88, 125427 (2013); M. Neupane, S. Xu, R. Sankar, N. Alidoust, G. Bian, C. Liu, I. Belopolski, T. R. Chang, H. T. Jeng, H. Lin, A. Bansil, F. Chou, M. Z. Hasan, arXiv:1309.7892 (2013); S. Borisenko, Q. Gibson, D. Evtushinsky, V. Zabolotnyy, B. Buechner, R. J. Cava, arXiv:1309.7978 (2013); Z. K. Liu, J. Jiang, B. Zhou, Z. J. Wang, Y. Zhang, Hongming Weng, D. Prabhakaran, SK. Mo, H. Peng, P. Dudin, T. Kim, M. Hoesch, Z. Fang, X. Dai, Z. X. Shen, D. L. Feng, Z. Hussain, Y. L. Chen, Nature Materials 13, 677 (2014).

[18] D. Thomas, J. Phys. Chem. Solids 15, 86 (1960).

[19] J. Hopfdeld, J. Phys. Chem. Solids 15, 97 (1960).

[20] M. D. Jones, R. C. Albers, Phys. Rev. B 79, 045107 (2009); L. Du, L. Huang, X. Dai, Eur. Phys. J. B 86, 94 (2013).

[21] P. Blaha, K. Schwarz, G. Madsen, D. Kvasnicka and J. Luitz, WIEN2k, An Augmented Plane Wave + Local Orbitals Program for Calculating Crystal Properties (2001), ISBN 3-9501031-1-2.

[22] N. Marzari and D. Vanderbilt, Phys. Rev. B 56, 12847 (1997); I. Souza, N. Marzari and D. Vanderbilt, Phys. Rev. B 65, 035109 (2001).

[23] A. D. Becke and E. R. Johnson, J. Chem. Phys.

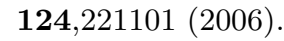

[24] HSE is implemented in VASP codes. G. Kresse and J. Hafner, Phys. Rev. B 47, 558 (1993); G. Kresse and D. Joubert, Phys. Rev. B 59, 1758 (1999); J. Heyd, G. Scuseria, and M. Ernzerhof, J. Chem. Phys. 118, 8207 (2003).

[25] Hongming Weng, Xi Dai and Zhong Fang, MRS Bulletin (in press).

[26] R. Yu, X. L. Qi, A. Bernevig, Z. Fang, and X. Dai, Phys. Rev. B 84, 075119 (2011); Alexey A. Soluyanov and David Vanderbilt, Phys. Rev. B 83, 235401 (2011).

[27] W. Zhang, R. Yu, W. Feng, Y. Yao, H. Weng, X. Dai, and Z. Fang, Phys. Rev. Lett. 106, 156808 (2011).

[28] H. J. Zhang, C. X. Liu, and S. C. Zhang, Phys. Rev. Lett. 111, 066801 (2013).

[29] Liang Fu and C. L. Kane, Phys. Rev. Lett. 100, 096407 (2008).

[30] B. Tell and P. M. Bridenbaugh, Phys. Rev. B 12, 3330 (1975); K. Yoodee, J. C. Woolley, V. Sa-yakanit, Phys. Rev. B 30, 5904 (1984).

[31] A. A. Burkov, L. Balents, Phys. Rev. Lett. 107, 127205 (2011); A. A. Burkov, M. D. Hook, L. Balents, Phys. Rev. B 84, 235126 (2011); G. B. Halász, L. Balents, Phys. Rev. B 85, 035103 (2012). 


\section{SUPPLEMENTAL MATERIAL}

\section{$\mathbb{Z}_{2}$ Indices by Wilson Loop Method}

Band inversion is a necessary condition for the existence of topological nontrivial states, but it is not sufficient to distinguish a topological insulator (TI), because topological invariant is a global character of the electronic structure in the whole Brillouin zone (BZ). The definition of parity criterion is convenient to identify TIs, but it can not be used in Wurtzite structure (or Zinc-blend) because of the lack of inversion symmetry. The Wilson loop method can be employed to study the evolution of Wannier function center [25, 26]. We calculated the Wannier center evolution for the six BZ plane of TIN, as shown in Fig 5 , and obtain $\mathbb{Z}_{2}$ indices as $(1 ; 000)$, demonstrating that TIN is a $3 \mathrm{D}$ strong TI.

\section{First-principles Energy Bands with lattice strain of $a=1.06 a_{0}$ and $c=1.10 c_{0}$.}

To tune the effective SOC parameter of TIN, we can enlarge the lattice constants to reduce the $p$ - $d$ hybridization. From realistic first-principles calculation, we found that if $a=a_{0}$ and $c \geq 1.150 c_{0}$ (or $c=1.10 c_{0}$ and $\left.1.04 a_{0} \leq a \leq 1.06 a_{0}\right)$ TIN would be in 3D Dirac semimetal state. The band structure of TIN with $a=1.06 a_{0}$ and $c=1.10 c_{0}$ is shown in Fig. 6 It is clear that there is a $3 \mathrm{D}$ Dirac point on $\Gamma-A$ path.

\section{Energy Bands of TIP and TlAs}

As TIN is a TI, it is natural to ask how about TIP and TlAs. We have found that the total energy of Wurtzite structure is lower than that of Zinc-blende structure for both of TIP and TlAs with relaxed structure. Band inversion occurs in both of them, but there is no finite band gap as shown in Fig.7. The effective SOC for TIP is nearly zero, because the intrinsic SOC of P-3p orbital is comparable to the $p$ - $d$ hybridization, which is in good agreement with model analysis in main text.

\section{Tight-binding Energy Bands by tuning effective SOC}

The phase diagram in Fig. 4 in main text is obtained by using the tight-binding model based on Wannnier functions with different effective SOC parameters. As shown in Fig, 8 it is a 3D Dirac semimetal when $\lambda_{e f f}>0$, otherwise it is a TI.
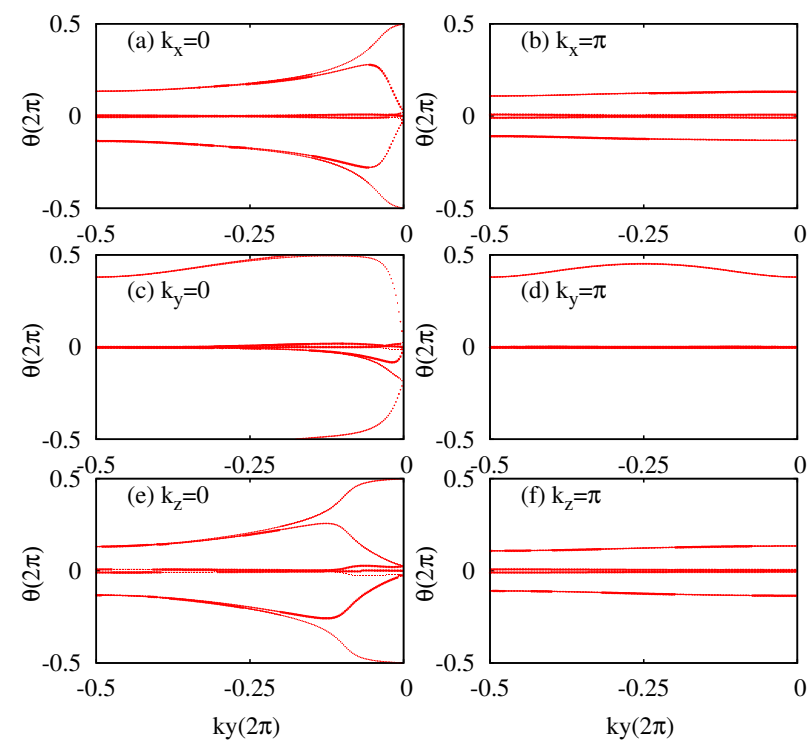

FIG. 5: (Color online) The Wannier function center evolution on BZ planes (a) $k_{x}=0$, (b) $k_{x}=\pi$, (c) $k_{y}=0$, (d) $k_{y}=\pi$, (e) $k_{z}=0$, and (f) $k_{z}=\pi$. It demonstrates the $\mathbb{Z}_{2}$ indices as $(1 ; 000)$.
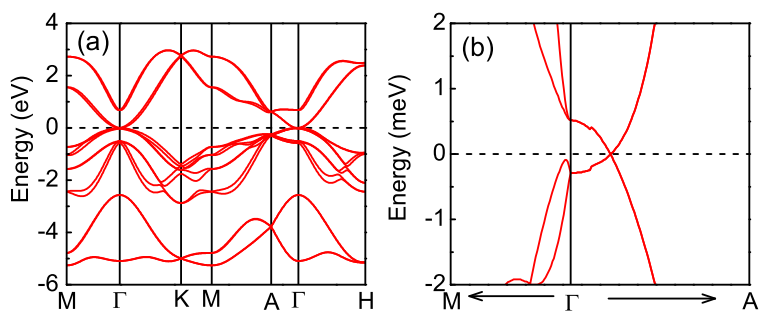

FIG. 6: (Color online) (a) The band structure of strained TIN with $a=1.06 a_{0}$ and $c=1.10 c_{0}$. (b)Partially enlarged view of (a).

\section{Kane Model}

The $8 \times 8$ Kane model can be used to describe the band structure of TIN. Firstly, a $4 \times 4$ model without SOC can be constructed in the basis set as $|S\rangle,\left|P_{+}\right\rangle,\left|P_{-}\right\rangle,\left|P_{z}\right\rangle$, where $\left|P_{ \pm}\right\rangle=\frac{1}{\sqrt{2}}\left(\left|P_{x}\right\rangle \pm i\left|P_{y}\right\rangle\right)$. The Hamiltonian reads

$H_{4 \times 4}(\vec{k})=\left(\begin{array}{cccc}E_{s} & i p_{1} k_{+} & i p_{1} k_{-} & d+i k_{z} p_{2} \\ -i p_{1} k_{-} & \lambda+\theta & a_{5} k_{-}^{2} & a_{6} k_{z} k_{-} \\ -i p_{1} k_{+} & a_{5} k_{+}^{2} & \lambda+\theta & a_{6} k_{z} k_{+} \\ d-i p_{2} k_{z} & a_{6} k_{z} k_{+} & a_{6} k_{z} k_{-} & \lambda-\delta\end{array}\right)$

The $8 \times 8$ model with $\mathrm{SOC}$ can be constructed as $H_{8 \times 8}(\vec{k})=I \otimes H_{4 \times 4}(\vec{k})+H_{s o} . \quad H_{s o}$ has the following form in the basis order: $\left|S_{\uparrow}\right\rangle,\left|P_{+\uparrow}\right\rangle,\left|P_{-\uparrow}\right\rangle,\left|P_{z \uparrow}\right\rangle,\left|S_{\downarrow}\right\rangle$, $\left|P_{+\downarrow}\right\rangle,\left|P_{-\downarrow}\right\rangle,\left|P_{z \downarrow}\right\rangle$. 

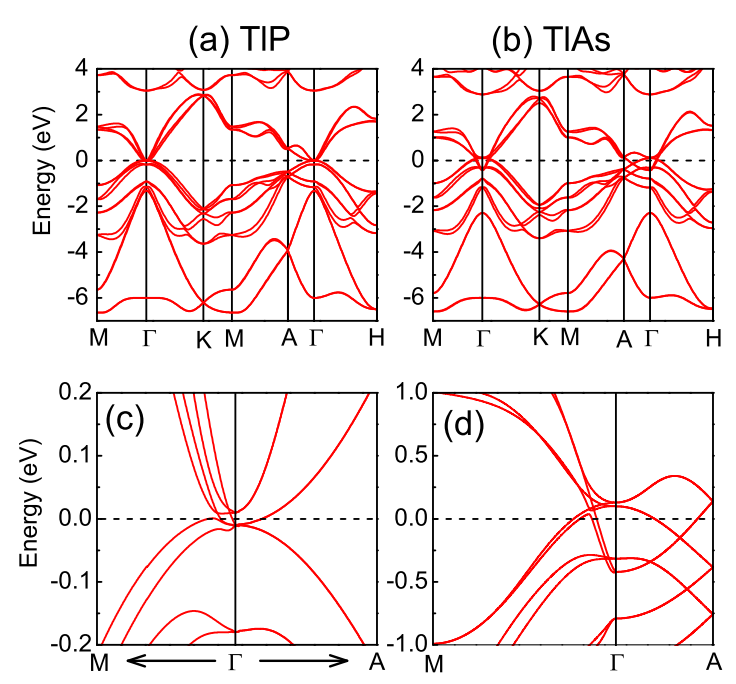

FIG. 7: (Color online) The band structure of (a) TIP and (b) TlAs. (c) and (d) are the partially enlarged view of (a) and (b), respectively.
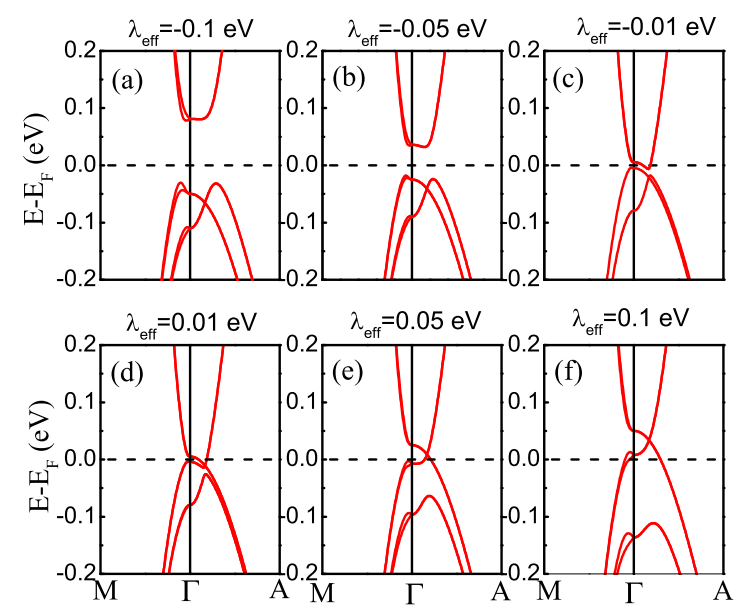

$H_{\Gamma}(\vec{k})=\epsilon_{0}(\vec{k})+\left(\begin{array}{cccc}M(\vec{k}) & i A k_{z} & D k_{-} & -i A k_{-} \\ -i A k_{z} & -M(\vec{k}) & -i A k_{-} & D k_{-} \\ D k_{+} & i A k_{+} & M(\vec{k}) & i A k_{z} \\ i A k_{+} & D k_{+} & -i A k_{z} & -M(\vec{k})\end{array}\right)$

FIG. 8: (Color online) The band structure of Wannier tightbinding model with effective SOC parameter as (a) $\lambda_{e f f}=-0.1$ $\mathrm{eV}$, (b) $\lambda_{e f f}=-0.05 \mathrm{eV}$, (c) $\lambda_{e f f}=-0.01 \mathrm{eV}$, (d) $\lambda_{e f f}=0.01 \mathrm{eV}$, (e) $\lambda_{e f f}=0.05 \mathrm{eV}$ and (f) $\lambda_{e f f}=0.1 \mathrm{eV}$.

$$
H_{s o}=\frac{\xi}{2}\left(\begin{array}{cccccccc}
0 & 0 & 0 & 0 & 0 & 0 & 0 & 0 \\
0 & 1 & 0 & 0 & 0 & 0 & 0 & 0 \\
0 & 0 & -1 & 0 & 0 & 0 & 0 & \sqrt{2} \\
0 & 0 & 0 & 0 & 0 & -\sqrt{2} & 0 & 0 \\
0 & 0 & 0 & 0 & 0 & 0 & 0 & 0 \\
0 & 0 & -\sqrt{2} & 0 & 0 & -1 & 0 & 0 \\
0 & 0 & 0 & 0 & 0 & 0 & 1 & 0 \\
0 & \sqrt{2} & 0 & 0 & 0 & 0 & 0 & 0
\end{array}\right)
$$

where $k_{ \pm}=k_{x} \pm i k_{y}$ and $M(\vec{k})=M_{0}-M_{1} k_{z}^{2}-$ $M_{2}\left(k_{x}^{2}+k_{y}^{2}\right)$ with parameters $M_{0}, M_{1}, M_{2}<0$ to reproduce band inversion. The parameter $\mathrm{D}$ is induced to describe the breaking of inversion symmetry. In such case, the energy dispersion is $E(\vec{k})=\epsilon_{0}(\vec{k}) \pm$ $\sqrt{M(\vec{k})^{2}+A^{2} \vec{k}^{2}+D^{2} k_{+} k_{-}+2 \sqrt{D^{2}\left(M^{2}+A^{2} k_{z}^{2}\right) k_{+} k_{-}}}$.

We can take the values as $M_{0}=-0.1, M_{1}=-10$, $M_{2}=-10, A=0.5$ and $D=0.1$, to study the low

energy physics qualitatively. written as the states with definite angular momentum $J$ and $J_{z}$, i. e., $\left|S_{J=\frac{1}{2}}, J_{z}= \pm \frac{1}{2}\right\rangle,\left|P_{\frac{3}{2}}, \pm \frac{3}{2}\right\rangle,\left|P_{\frac{3}{2}}, \pm \frac{1}{2}\right\rangle$, $\left|P_{\frac{1}{2}}, \pm \frac{1}{2}\right\rangle$. In Wurtzite TIN, the valence band is mainly contributed by $\left|P_{\frac{1}{2}}, \pm \frac{1}{2}\right\rangle$, and inverted states is $\left|S_{\frac{1}{2}}, \pm \frac{1}{2}\right\rangle$. Therefore, the inversion mechanism can be described by the $\left|P_{\frac{1}{2}}, \pm \frac{1}{2}\right\rangle$ and the $\left|S_{\frac{1}{2}}, \pm \frac{1}{2}\right\rangle$ states. We can construct a low energy Hamiltonian around $\Gamma$ point, by considering only the minimal basis set of $\left|S_{\frac{1}{2}}, \frac{1}{2}\right\rangle,\left|P_{\frac{1}{2}}, \frac{1}{2}\right\rangle,\left|S_{\frac{1}{2}},-\frac{1}{2}\right\rangle$ and $\left|P_{\frac{1}{2}},-\frac{1}{2}\right\rangle$ states. We can directly get it by downfolding the 8 -band model into the subspace spanned by the 4 mimimal basis. The resulting $H_{\Gamma}(\vec{k})$ reads,

where $E_{s}=E_{s}+s_{1} k_{z}^{2}+s_{2} k_{+}^{2}, \lambda=E_{p}+a_{1} k_{z}^{2}+$ $a_{2} k_{+}^{2}, \theta=a_{3} k_{z}^{2}+a_{4} k_{+}^{2}$. The parameters take val-
ues from fitting first-principles results as $E_{s}=-2.05842$, $E_{p}=0.00015, \delta=0.07903, s_{1}=8.0, s_{2}=3.0, a_{1}=-2.0, a_{2}=-$ $5.3344, a_{3}=0.0, a_{4}=0.1, \quad a_{5}=0.3, \quad a_{6}=0.2, \quad p_{1}=2.9698$, $p_{2}=6.3, \xi=-0.025$. It is noticed that the effective SOC parameter $\xi$ is negative. 